\title{
New Data on Access to Mental Health and Addictions Services and Home and Community Care
}

\author{
Maria Zaccaria Cho, Jennifer Frood, Liudmila Husak, Jeanie Lacroix, Norma Hall and Luke Andrew Turcotte
}

\begin{abstract}
As the population ages, more Canadians need home care to help manage their health conditions and live safely at home. For Canadians of all ages, timely access to mental health and addictions services is an area of growing concern. The impact of the COVID-19 pandemic and its strain on health system resources have further highlighted the need to improve services in these areas. The Canadian Institute for Health Information (CIHI) is working with governments across Canada to bridge data gaps, develop indicators and publicly report results as part of a collective effort to improve access in these two sectors. Results for three new indicators were released by $\mathrm{CIHI}$ in 2020.
\end{abstract}

\section{Introduction}

Canadians now have more information about access to home and community care and to mental health and addictions services. Results are available for three new indicators chosen by the federal ${ }^{1}$,provincial and territorial health ministries, in consultation with Canadians, to measure access in these sectors (Government of Canada 2017). Initial results provide a baseline against which to track improvements. Over time, these indicators will tell a clearer story about access to care across the country, identify where there are gaps in services and help make meaningful changes to improve the experiences of Canadian patients and their families.

\section{Key Findings}

On August 6, 2020, the Canadian Institute for Health Information (CIHI) released results, based on 2018-2019 data, for three new indicators (CIHI 2020):

- Self-harm, including suicide. In 2018, 25,000 Canadians were hospitalized or died due to intentional self-harm.

- Caregiver distress. Ninety-six percent of individuals receiving long-term home care had an unpaid caregiver. More than one in three of these caregivers were distressed.

- New long-term care residents who potentially could have been cared for at home. About one in nine newly admitted longterm care residents potentially could have been cared for at home.
The data predate the COVID-19 pandemic in 2020. While the extent of the impact of COVID-19 on access to mental health and addictions services and to home and community care is not yet known, CIHI's data represent a baseline from which progress can be measured over time. Detailed results, as well as more information on data sources, data limitations and methodology, are available at https:/www.cihi.ca/en/ measuring-access-to-priority-health-services.

\section{Self-Harm, Including Suicide}

This indicator measures the rate of hospital stays for and deaths from intentional self-harm, regardless of suicidal intent. Even when there is no intention to die, deliberate self-harm signals emotional distress that may end in accidental death or serious injury.

In 2018-2019, almost 25,000 Canadians stayed in hospital after intentionally harming themselves or died by suicide. This is the equivalent of almost 70 self-harm events every day. Of these nearly 25,000 Canadians who harmed themselves, more than 3,800 died as a result of their injuries. These deaths were much more likely to occur in the community (89\%) than in the hospital. Rates of self-harm, including suicide, varied more than seven-fold among provinces and territories.

Hospitalization rates for self-harm were higher for females than males across most age groups, with the highest rates seen in young women. Rates were three times higher for girls and women of 10-24 years than for males in the same age group.

The picture looks different, however, when looking at deaths from self-harm. Suicide rates were higher for males, affecting men of 45-64 years the most (Figure 1).

Self-harm impacts Canadians of all income levels. However, Canadians living in the lowest-income neighbourhoods had twice the rate of hospital stays compared to those living in the highest-income neighbourhoods.

Individuals living in rural or remote areas were also more likely to have a hospital stay for self-harm compared to those in urban areas. 
FIGURE 1.

Key findings for self-harm, including suicide

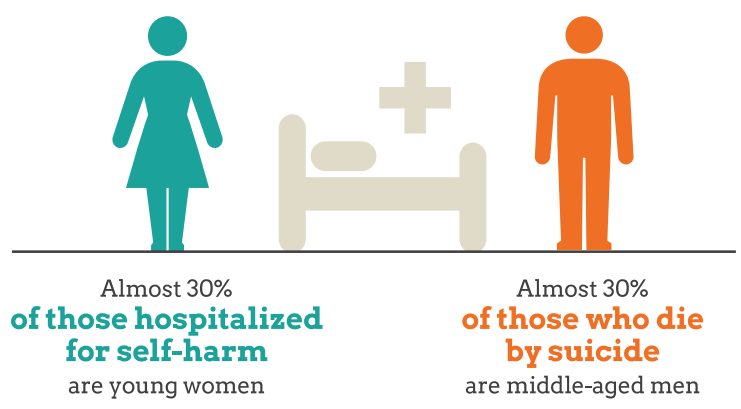

Source: Hospital Morbidity Database and Ontario Mental Health Reporting System 2018-2019, Canadian Institute for Health Information; Canadian Vital Statistics, Death Database 2018, Statistics Canada; and Yukon Bureau of Statistics 2018

\section{Caregiver Distress}

This indicator measures the proportion of unpaid caregivers who experience distress while caring for a family member or friend who receives publicly funded homecare services and supports.

Unpaid caregivers play a vital role in our health systems by supporting people with health challenges to stay at home or in the community. Providing care to a family member or friend can be demanding, with profound emotional, mental, financial and physical impacts.

In 2018-2019, 96\% of individuals who received long-term home care had an unpaid caregiver. More than one in three of these caregivers of individuals who received home care in reporting provinces and territories were distressed, which can include feelings of anger or depression or the inability to continue with caring activities.

Caregivers who are distressed spend an average of 38 hours a week providing care - the equivalent of a full-time job. In comparison, the data show that this is twice the number of caregiving hours provided by caregivers who are not distressed (19 hours a week; Figure 2).

FIGURE 2.

Key findings for caregiver distress

\section{Caregivers in distress spend the equivalent of a full-time job providing care}

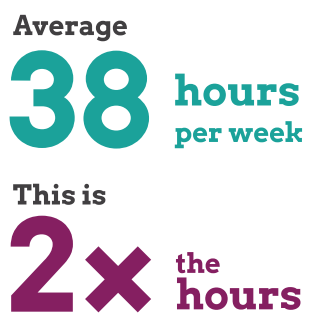

provided by caregivers who are not distressed

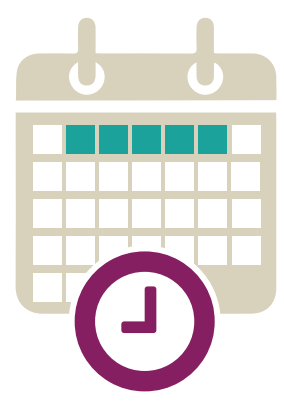

Source: Home Care Reporting System 2018-2019, Canadian Institute for Health Information
Among caregivers who are distressed, seven out of 10 supported individuals who required substantial assistance with personal care activities, including bathing or showering, eating, dressing, brushing their teeth, toilet use and getting in and out of bed. Caregivers were also twice as likely to be distressed if they cared for a person with communication difficulties or behavioural problems.

\section{New Long-Term Care Residents Who Potentially Could Have Been Cared for at Home}

Most older adults in Canada want to remain at home for as long as possible as they age. However, some individuals are admitted to long-term care even though they potentially could have been cared for at home with proper supports. These include individuals with mild cognitive impairment who require no more than limited assistance with activities of daily living. This indicator measures the percentage of people who were newly admitted to long-term care and who had similar health characteristics to people living well at home with formal supports in place.

In 2018-2019, 45,000 Canadians were admitted to a longterm care facility in reporting provinces and territories. Of these residents, one in nine potentially could have been cared for at home with formal supports in place. This represents more than 5,000 long-term care spaces across Canada (Figure 3).

Where people live can influence their likelihood of entering long-term care. People who lived in rural areas were over 50\% more likely than those living in urban areas to be admitted to long-term care when they potentially could have been cared for at home. This may be due to fewer homecare services being offered in rural and remote areas.

Similar to other newly admitted long-term care residents, two in three were mostly women and over $50 \%$ were more than 85 years old.

Residents and family members of residents admitted to long-term care told us they experienced various barriers to remaining at home. A qualitative analysis of focus group discussions uncovered four overarching themes:

- Difficulty navigating the healthcare system. People experienced confusion and challenges around whom to contact, what services were available, the amount of time required to coordinate services and the lack of continuity across the system.

- Financial barriers. Because publicly funded home care does not cover all costs associated with caring for someone at home, some families experienced significant out-of-pocket expenses. Those living in rural and remote communities faced higher travel costs for medical appointments and limited availability of homecare services and supports.

- Responsiveness. People emphasized the importance of reliable homecare staff, as well as the need for services that were flexible to the changing needs of the person receiving care. 
- Access to special services. People highlighted the need for social and emotional support, help with non-medical needs and services tailored to their language and cultural needs.

FIGURE 3.

Key findings for residents newly admitted to long-term care

About 1 in 9

newly admitted

long-term care

residents potentially

could have been
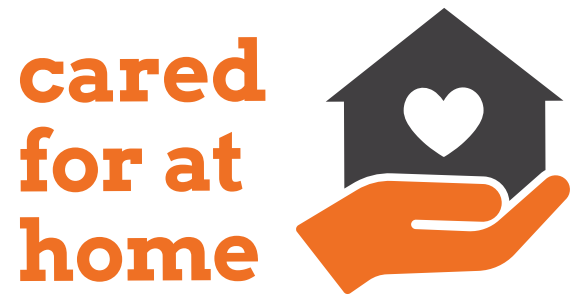

Source: Continuing Care Reporting System 2018-2019, Canadian Institute for Health Information

\section{Moving Forward}

These new indicators provide a baseline against which to track improvements in access to mental health and addictions services, and to home and community care over time. With annual reporting of results among a suite of pan-Canadian indicators, the indicators will tell us more about access to care in these areas, identify where there are gaps in services and help make meaningful changes to improve the experiences of Canadian patients and their families.

CIHI will continue to work with provincial and territorial governments, Health Canada, Statistics Canada and other partners to enhance and develop common information standards, improve data coverage and quality and explore new sources of data for public reporting (Government of Canada 2019). These include working closely with jurisdictions to monitor the impact of the COVID-19 pandemic and to help address the resulting data gaps.

\section{Note}

1. The federal government agreed to an asymmetrical arrangement with Quebec, distinct from the agreement referenced here.

\section{References}

Canadian Institute for Health Information (CIHI). 2020, August 2. Common Challenges, Shared Priorities: Measuring Access to Home and Community Care and to Mental Health and Addictions Services in Canada. Volume 2. Retrieved October 6, 2020. <https://www.cihi.ca/ sites/default/files/document/common-challenges-shared-priorities-vol2-report-en.pdf>.

Government of Canada. 2017. A Common Statement of Principles on Shared Health Priorities. Retrieved October 6, 2020. <https:// www.canada.ca/content/dam/hc-sc/documents/corporate/ transparency_229055456/health-agreements/principles-shared-healthpriorities.pdf $>$.

Government of Canada. 2019, August 13. Shared Health Priorities. Retrieved October 6, 2020. <https://www.canada.ca/en/health-canada/ corporate/transparency/health-agreements/shared-health-priorities. html>.

\section{About the Authors}

Maria Zaccaria Cho, BA (Hons), is a senior coordinator at $\mathrm{ClHI}$ in Toronto, ON. She can be reached by e-mail at mzaccariacho@cihi.ca.

Jennifer Frood, BA (Hons), MSc, is a project lead at $\mathrm{CIHI}$ in Toronto, ON.

Liudmila Husak, MD, MPH, is a manager at $\mathrm{CIHI}$ in Toronto, ON. Jeanie Lacroix, MPA, is a manager at $\mathrm{CIHI}$ in Toronto, ON

Norma Hall, $\mathrm{PhD}$, is a methodologist at $\mathrm{ClHI}$ in Ottawa, $\mathrm{ON}$.

Luke Andrew Turcotte, $\mathrm{PhD}$, is a postdoctoral fellow at $\mathrm{ClHI}$ in Ottawa, ON. He is supported by a Canadian Institutes of Health Research Health System Impact Fellowship.

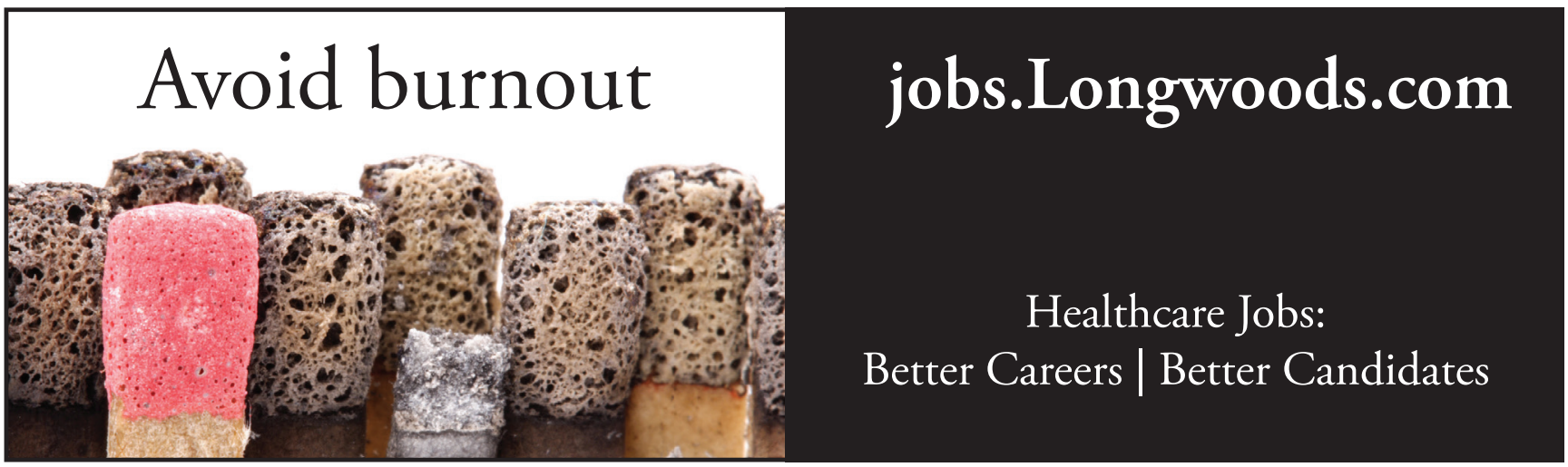

